\title{
Platelet hyperaggregability in high-fat fed rats: A role for intraplatelet reactive-oxygen species production
}

\author{
Priscila F Monteiro ${ }^{1}$, Rafael P Morganti ${ }^{1}$, Maria A Delbin², Marina C Calixto ${ }^{1}$, Maria E Lopes-Pires ${ }^{1}$, Sisi Marcondes ${ }^{1}$, \\ Angelina Zanesco ${ }^{2}$ and Edson Antunes ${ }^{1 *}$
}

\begin{abstract}
Background: Adiposity greatly increases the risk of atherothrombotic events, a pathological condition where a chronic state of oxidative stress is reported to play a major role. This study aimed to investigate the involvement of (NO)-soluble guanylyl cyclase (sGC) signaling pathway in the platelet dysfunction from high fat-fed (HFF) rats.

Methods: Male Wistar rats were fed for 10 weeks with standard chow (SCD) or high-fat diet (HFD). ADP (10 $\mu M$ )and thrombin $(100 \mathrm{mU} / \mathrm{ml})$-induced washed platelet aggregation were evaluated. Measurement of intracellular levels of ROS levels was carried out using flow cytometry. Cyclic GMP levels were evaluated using ELISA kits.

Results: High-fat fed rats exhibited significant increases in body weight, epididymal fat, fasting glucose levels and glucose intolerance compared with SCD group. Platelet aggregation induced by ADP $(n=8)$ and thrombin from HFD rats $(n=8)$ were significantly greater $(P<0.05)$ compared with SCD group. Platelet activation with ADP increased by $54 \%$ the intraplatelet ROS production in HFD group, as measured by flow cytometry $(n=6)$. Nacetylcysteine (NAC; $1 \mathrm{mM})$ and PEG-catalase $(1000 \mathrm{U} / \mathrm{ml})$ fully prevented the increased ROS production and platelet hyperaggregability in HFD group. The NO donors sodium nitroprusside (SNP; $10 \mu M)$ and SNAP (10 $\mu M)$, as well as the NO-independent soluble guanylyl cyclase stimulator BAY 41-2272 (10 $\mu \mathrm{M})$ inhibited the platelet aggregation in HFD group with lower efficacy $(P<0.05)$ compared with SCD group. The CGMP levels in response to these agents were also markedly lower in HFD group $(P<0.05)$. The prostacyclin analogue iloprost $(1 \mu \mathrm{M})$ reduced platelet aggregation in HFD and SCD rats in a similar fashion $(n=4)$.
\end{abstract}

Conclusions: Metabolic abnormalities as consequence of HFD cause platelet hyperaggregability involving enhanced intraplatelet ROS production and decreased NO bioavailability that appear to be accompanied by potential defects in the prosthetic haem group of soluble guanylyl cyclase.

Keywords: Platelet aggregation, Obesity, Reactive-oxygen species, Nitric oxide, Cyclic GMP, BAY 41-2272

\section{Background}

Platelets play an important physiological function in haemostasis system in response to vascular injury by preventing hemorrhage [1]. Effective platelet adhesion and aggregation require the synergistic contribution of multiple receptor-ligand interactions that transmit activating signals initiating a range of platelet biochemical and morphological responses, linked to cytoskeleton

\footnotetext{
* Correspondence: edson.antunes@uol.com.br

'Department of Pharmacology, Faculty of Medical Sciences, University of

Campinas (UNICAMP), Campinas, Sao Paulo, Brazil

Full list of author information is available at the end of the article
}

remodeling, granule secretion and the generation and release of endogenous soluble agonists, such as ADP and thromboxane $\mathrm{A}_{2}\left(\mathrm{TXA}_{2}\right)$ [2-5].

Endothelial cell-derived nitric oxide (NO) exerts an inhibitory effect in the platelet function by activation of cGMP/PKG pathway, which in turn leads to reduction in concentration of $\mathrm{Ca}^{2+}$, thus preventing adhesion and aggregation of platelets to the vascular wall [6]. Nonetheless, endothelium dysfunction, present in certain pathological conditions, is characterized by a decreased NO bioavailability which incites abnormal platelet activation leading to vascular thrombosis $[7,8]$. Platelet 
dysfunction is considered an end stage of cardiovascular complications in type II diabetes mellitus, obesity and atherosclerosis that results in clinical outcomes such as myocardial infarction, stroke and peripheral artery disease [9].

Obesity is an important public health problem affecting all ages and socioeconomic groups greatly elevating the incidence of cardiovascular and endocrine-metabolic disorders. A chronic state of oxidative stress and inflammation are the hallmark of adiposity that play a pivotal role in the physiopathological events in this disorder $[10,11]$. These pro-inflammatory and pro-oxidant effects are associated with increased reactive-oxygen species (ROS) production and decreased NO bioavailability, which increases the risk of athero-thrombotic events [12]. Nonetheless, the exact mechanisms by which adiposity induces platelet dysfunction remain poorly investigated. In addition, most of fatal cardiovascular events as consequence of thrombotic complication are not associated with complete vascular stenosis, but rather with alterations of pro-inflammatory and pro-oxidant biomarkers, which can predict future cardiovascular events. We hypothesized that intraplatelet ROS production in adiposity contributes to thrombotic events in endocrine-metabolic disorders. Therefore, we have investigated the ex-vivo platelet reactivity in response to ADP and thrombin in high fat-fed rats, and the involvement of platelet-derived ROS and NO-cGMP pathway in modulating the platelet reactivity.

\section{Methods}

\section{Animals and high-fat diet}

The experimental protocols were approved by the Ethical Principles in Animal Research adopted by the Brazilian College for Animal Experimentation (COBEA) and performed in compliance with the ARRIVE guidelines on animal research [13]. Male Wistar rats were housed in temperature-controlled rooms on a $12 \mathrm{~h}$ light-dark cycle. The animals were housed two per cage and fed for 10 weeks with either a standard chow diet (carbohydrate: $70 \%$; protein: $20 \%$; fat: $10 \%$ ) or a high-fat diet that induces obesity (carbohydrate: 29\%; protein: 16\%; fat: $55 \%$ ), according to our previous work [14].

\section{Body Weight, epididymal fat mass and glycemia}

The body weight and epididymal fat mass were evaluated in the beginning and at final time of the study. The glucose concentration was measured in blood from the tail vein (Accu-Check Performa, Roche Diagnostics, Indianapolis, IN, USA).

\section{Oral glucose tolerance test (OGTT) and insulin tolerance test (ITT)}

Oral glucose tolerance test was performed after $12 \mathrm{~h}$ of fasting. Control and high-fat fed (HFF) obese rats received a $20 \%$ glucose solution $(2 \mathrm{~g} / \mathrm{kg})$ by gavage. Blood samples were collected from tail vein at basal condition and after 30, 60 and 120 min of glucose loading. Wholebody insulin sensitivity was analyzed by the Insulin Tolerance Test (ITT). Venous blood samples were collected before $(0 \mathrm{~min})$ and 15,30 and $60 \mathrm{~min}$ after an intraperitoneal injection of regular insulin $(0.75 \mathrm{U} / \mathrm{kg})$.

\section{Isolation of blood platelets and aggregation assays}

Rats were anaesthetized with isoflurane, and blood was collected from abdominal aorta in 1:9 (v/v) of ACD-C (12.4 mM sodium citrate, $13 \mathrm{mM}$ citric acid, $11 \mathrm{mM}$ glucose). Platelet-rich plasma (PRP) was obtained by centrifugation of whole blood at $200 \mathrm{~g}$ for $15 \mathrm{~min}$ at room temperature. Five milliliters of PRP were added to $7 \mathrm{ml}$ of washing buffer $(140 \mathrm{mM} \mathrm{NaCl}, 0.5 \mathrm{mM} \mathrm{KCl}, 12$ $\mathrm{mM}$ trisodium citrate, $10 \mathrm{mM}$ glucose, $12.5 \mathrm{mM}$ saccharose, pH6), and centrifuged (800 g, $13 \mathrm{~min})$. The pellet was resuspended in washing buffer, and the procedure was repeated once. The platelets were gently suspended in Krebs solution $(118 \mathrm{mM} \mathrm{NaCl}, 25 \mathrm{mM}$ $\mathrm{NaHCO}_{3}, 1.2 \mathrm{mM} \mathrm{KH} \mathrm{PO}_{4}, 1.7 \mathrm{mM} \mathrm{MgSO} 4,5.6 \mathrm{mM}$ glucose, $\mathrm{pH}$ 7.4). The platelet number was adjusted to $1.2 \times 10^{8}$ platelets $/ \mathrm{ml}$ in the presence of $1 \mathrm{mM} \mathrm{CaCl}_{2}$. Platelet aggregation was measured in a two channel aggregometer (Chronolog Lumi-Aggregometer model 560-Ca, Havertown, PA, USA) at $37^{\circ} \mathrm{C}$ with stirring (1000 rpm). Platelet aggregation assays were carried using $\operatorname{ADP}(10 \mu \mathrm{M})$ or thrombin $(100 \mathrm{mU} / \mathrm{ml})$.

\section{Measurement of reactive-oxygen species (ROS) by flow cytometry}

Measurement of intracellular levels of ROS was carried out according to a previous study [15]. Briefly, washed platelets (obtained as detailed above) were resuspended in Krebs-Ringer solution at $1.2 \times 10^{8}$ platelet $/ \mathrm{mL}$ in the presence of $1 \mathrm{mM}$ of calcium and $5 \mu \mathrm{M}$ of 2'-7'-dichlorofluorescin diacetate (DCFH-DA). Platelet suspension $(500 \mu \mathrm{L})$ were pre-incubated with $\mathrm{N}$-acetylcysteine (NAC, $1 \mathrm{mM})$ or PEG-catalase $(1000 \mathrm{U} / \mathrm{ml})$ for $15 \mathrm{~min}$ before addition of DCFH-DA. Platelet suspension was then incubated or not with ADP $(20 \mathrm{M})$ or $\mathrm{H} 2 \mathrm{O} 2(8$ $\mathrm{mM}$; positive control) for $20 \mathrm{~min}$. Platelet samples were then centrifuged ( $800 \mathrm{~g} 10 \mathrm{~min}$ ), and the pellet was resuspended in Krebs solution $(500 \mu \mathrm{L})$. Samples were analyzed using a Becton Dickinson flow cytometer (FACSCalibur, Becton Dickinson, San José, CA, USA) equipped with a $488 \mathrm{~nm}$ wavelength argon laser, $510 \times$ $540 \mathrm{~nm}$ band pass filters. Platelets were identified by the forward and side scatter signals. Ten thousand platelet specific events were initially analyzed by the cytometer. Non-activated and activated platelets were gated so as not to analyze platelet aggregates and microparticles. The gates were then analyzed for mean fluorescence. 


\section{Extraction and measurement of cGMP}

Washed platelets $\left(1.2 \times 10^{8}\right.$ platelets $\left./ \mathrm{mL}\right)$ were incubated with the phosphodiesterase inhibitor 3-isobutyl-1methyl-xanthine (IBMX; $2 \mathrm{mM}$ ) for $15 \mathrm{~min}$. Next, platelets were incubated with sodium nitroprusside (SNP, 10 $\mu \mathrm{M}), \mathrm{S}$-nitroso-N-acetylpenicillamine (SNAP, $10 \mu \mathrm{M}$ ) or BAY 41-2272 $(10 \mu \mathrm{M})$ for $3 \mathrm{~min}$, after which the reaction was interrupted by the addition of cold-acidified absolute ethanol (67\%, vol/vol), and samples were vigorously agitated for $30 \mathrm{~s}$. Cell samples were centrifuged $\left(4,000 \mathrm{~g}, 30 \mathrm{~min}\right.$ at $\left.4^{\circ} \mathrm{C}\right)$. Supernatants were dried at 55$60^{\circ} \mathrm{C}$ under a stream of nitrogen. Cyclic GMP was measured by using a kit from Cayman Chemical (Ann Arbor, MI). The assays were performed in duplicates. The limit of cGMP detection is $1 \mathrm{pmol} / \mathrm{mL}$.

\section{Materials}

Adenosine diphosphate (ADP), thrombin, PEG-catalase, $\mathrm{N}$-acetylcysteine (NAC), sodium nitroprusside, Snitroso-N-acetylpenicillamine (SNAP) were purchased from Sigma Chem. Co. (St. Louis, MO, USA). 5-cyclopropyl-2-[1-(2-fluoro-benzyl)-1H-pyrazolo[3,4-b]pyridin3-yl]-pyrimidin-4-ylamine (BAY 41-2272) was provided by Pharma Research Center, Bayer (Wuppertal, Germany). Iloprost was supplied by Schering (Germany).

\section{Statistical analysis}

Data are expressed as means \pm SEM of $n$ rats. The statistical significance between groups was determined by using one-way ANOVA followed by the Bonferroni test. Where appropriate, unpaired Student's t test was used to compare specific groups. Significance was established at $P<0.05$.
Table 1 Effect of high-fat diet on body weight, epididymal fat weight and glucose levels

\begin{tabular}{lll}
\hline & SCD & HFD \\
\hline Body weight $(\mathrm{g})$ & $457 \pm 8.1$ & $551 \pm 17^{*}$ \\
Epididymal fat $(\mathrm{g})$ & $7.6 \pm 0.6$ & $17.9 \pm 1.5^{*}$ \\
Glucose $(\mathrm{mg} / \mathrm{dl})$ & $89 \pm 3.8$ & $127 \pm 3.2^{*}$ \\
\hline
\end{tabular}

Male Wistar rats were fed with either a standard chow diet (SCD) or high-fat diet (HFD) during 10 weeks.

Values represent means \pm SEM animal for $n=4-6$. ${ }^{*} P<0.05$ compared with control group.

\section{Results}

Body weight, oral glucose tolerance test (OGTT) and insulin tolerant test (ITT)

Rats treated with high-fat diet (HFD) exhibited a significant increase in body weight and epididymal fat compared with animals receiving standard-chow diet (SCD), approximately $20 \%$ and $135 \%(P<0.05)$, respectively (Table 1). Fasting glucose levels were significantly higher in HFD compared with SCD group, about of $40 \%(P<$ 0.05). The OGTT showed that glucose levels remained increased after glucose consumption in HFD in all evaluated time-points in comparison with SCD group (Figure $1 \mathrm{~A} ; n=4-6)$. Insulin sensitivity was markedly reduced in HFD compared with SCD rats $(P<0.05)$, as assessed by the ITT (Figure 1B).

ADP- and thrombin-induced washed platelet aggregation Platelet aggregation induced by ADP $(10 \mu \mathrm{M})$ was significantly greater $(P<0.05)$ in washed platelets obtained from HFD compared with SCD group $(80.0 \pm 1.1 \%$ and $59.2 \pm 1.5 \%$, respectively; $n=8)$. Similarly, platelet aggregation induced by thrombin $(100 \mathrm{mU} / \mathrm{ml})$ was

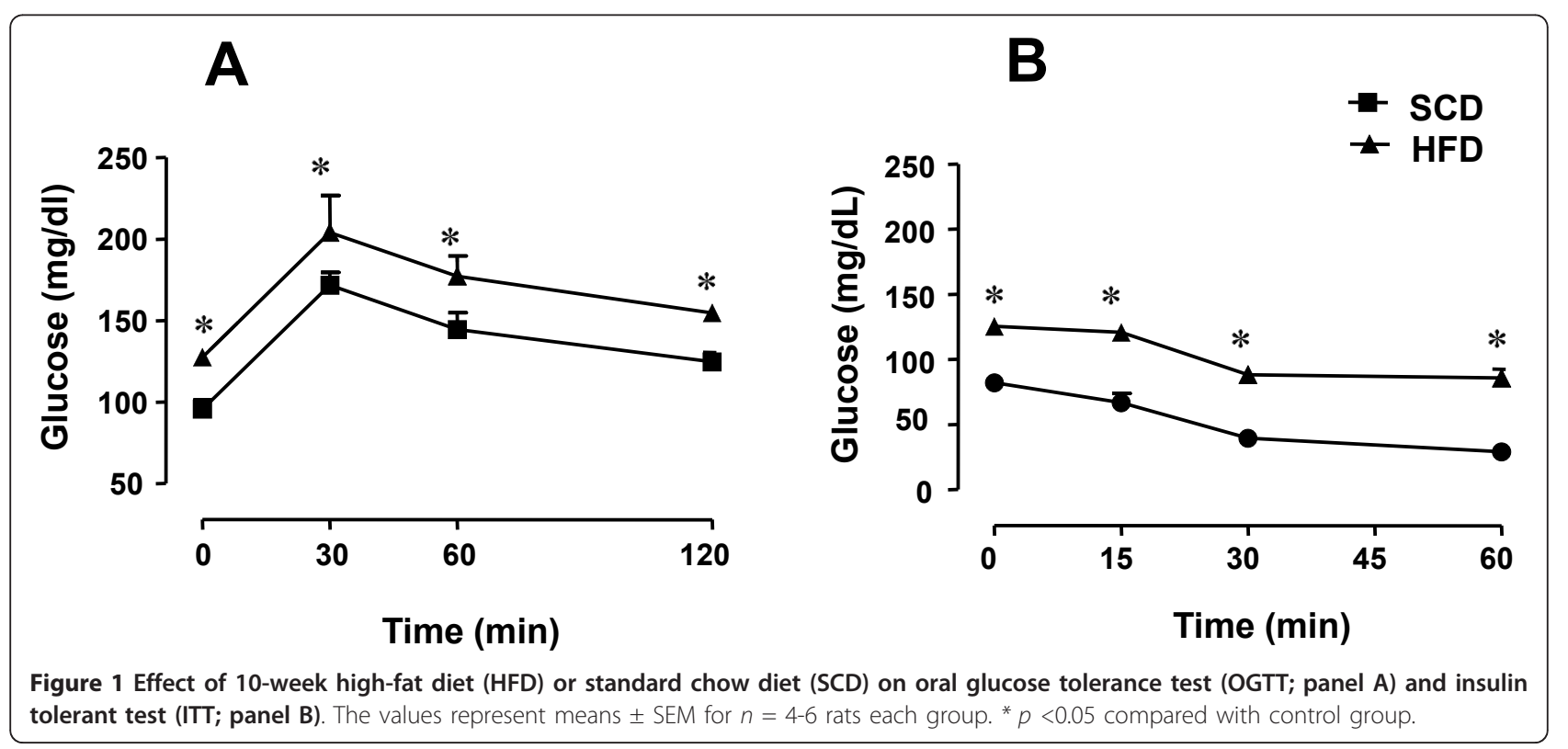


significantly greater $(P<0.05)$ in washed platelets obtained from HFD compared with SCD group (81.2 \pm $3.4 \%$ and $60.2 \pm 3.1 \%$, respectively; $n=8$ ).

\section{Generation of ROS in washed platelets}

The basal production of intraplatelet ROS did not significantly change between HFD and SCD groups (Figure 2). In SCD group, activation of platelets with ADP (10 $\mu \mathrm{M})$ did not significantly change the ROS production compared with its respective basal ROS production. However, in HFD group a marked increase in the intraplatelet ROS production was found after activation by ADP, by about of $54 \%(P<0.001$; Figure 2$)$. Pretreatment of platelets with NAC $(1 \mathrm{mM})$ or PEG-catalase $(1000 \mathrm{U} / \mathrm{ml})$ did not significantly affect the ROS production in SCD, but prevented the increased ROS production in platelets from HFD rats $(n=6$ each group; Figure 2).

\section{Effect of NAC and PEG-catalase on platelet hyperaggregability of HFF Rats}

Pretreatment of platelets with NAC (1 mM, $3 \mathrm{~min})$ or PEG-catalase $(1000 \mathrm{U} / \mathrm{ml}, 3 \mathrm{~min})$ did not significantly affect thrombin- or ADP-induced platelet aggregation in SCD rats $(n=4-7$; Figure 3$)$. However, NAC and PEGcatalase fully prevented the platelet hyperaggregability induced by thrombin or ADP in HFD group (Figure 3).

Effect of sodium nitroprusside (SNP), S-nitroso-Nacetylpenicillamine (SNAP) and BAY 41-2271 on platelet aggregation and CGMP intracellular levels In SCD rats, ADP-induced platelet aggregation was largely reduced by prior incubation with the NO donors
SNP $(10 \mu \mathrm{M} ; n=4)$ and SNAP $(10 \mu \mathrm{M} ; n=5)$, as well as by the NO-independent soluble guanylyl cyclase stimulator BAY 41-2272 (10 $\mu \mathrm{M} ; n=7)$, as shown in Figure 4A. The reduction of platelet aggregation by SNP, SNAP and BAY 41-2272 were accompanied by 7.0-, 7.6and 12.3 -fold increase $(P<0.001)$ in the amounts of intracellular cGMP levels, respectively (Figure 4B). In HFD rats, inhibition of ADP-induced platelet aggregation by SNP, SNAP and BAY-412272 was markedly lower compared with SCD group ( $n=4$ each group; Figure 4A). In addition, in HFD rats, SNP and SNAP did not significantly increase the amounts of cGMP above baseline (Figure 4B). The increases in cGMP by BAY-412272 were also significantly lower in HFD compared with SCD rats (Figure 4B). Incubation of platelets with the soluble guanylyl cyclase inhibitor ODQ (10 $\mu \mathrm{M}, 10 \mathrm{~min}$ ) before addition of SNP, SNAP and BAY 41-2272 (10 $\mu \mathrm{M}$ each) abolished the increase in the cGMP levels ( $n=3$; not shown).

\section{Effect of iloprost on platelet aggregation}

Pretreatment of platelets with iloprost $(1 \mu \mathrm{M}, 3 \mathrm{~min})$, a prostacyclin analogue that acts directly in cAMP/PKA signaling pathway, nearly abolished the ADP-induced platelet aggregation, as observed in both SCD and HFD rats $(n=4$; Table 2). No statistical differences were found between control and HFF rats.

\section{Discussion}

The present study shows that rats fed with high-fat diet (HFD) exhibit ex-vivo platelet hyperaggregability to ADP and thrombin, which is accompanied by higher intraplatelet ROS production. The platelet hyperaggregability
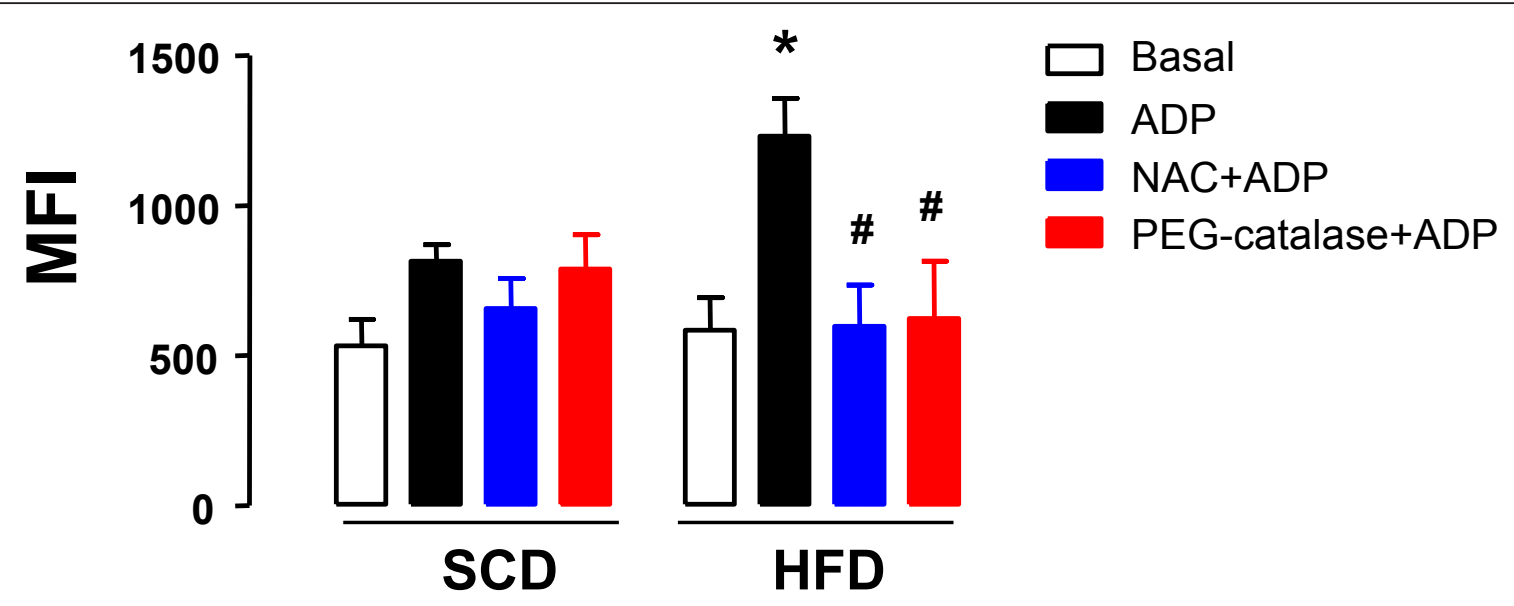

Figure 2 Effect of high-fat diet in the generation of intraplatelet reactive-oxygen species (ROS). Male Wistar rats were fed with either a standard chow diet (SCD) or high-fat diet (HFD) during 10 weeks. Washed platelets $\left(1.2 \times 10^{8}\right.$ platelets $\left./ \mathrm{ml}\right)$ from SCD or HFF rats were preincubated with $\mathrm{N}$-acetylcysteine (NAC, $1 \mathrm{mM}$ for $15 \mathrm{~min}$ ) or PEG-catalase (1000 mU/ml, $15 \mathrm{~min}$ ) and then stimulated with ADP (10 $\mu$ M). Generation of ROS was quantified by flow cytometry using 2'-7'-dichloroflurescin diacetate (DCFH-DA). Results are shown as mean \pm SEM values for $n=6-7$. ${ }^{*} P<0.05$ compared with SCD group. ${ }^{\#} P<0.05$ compared with ADP in HFF group. MFI = mean fluorescence index. 

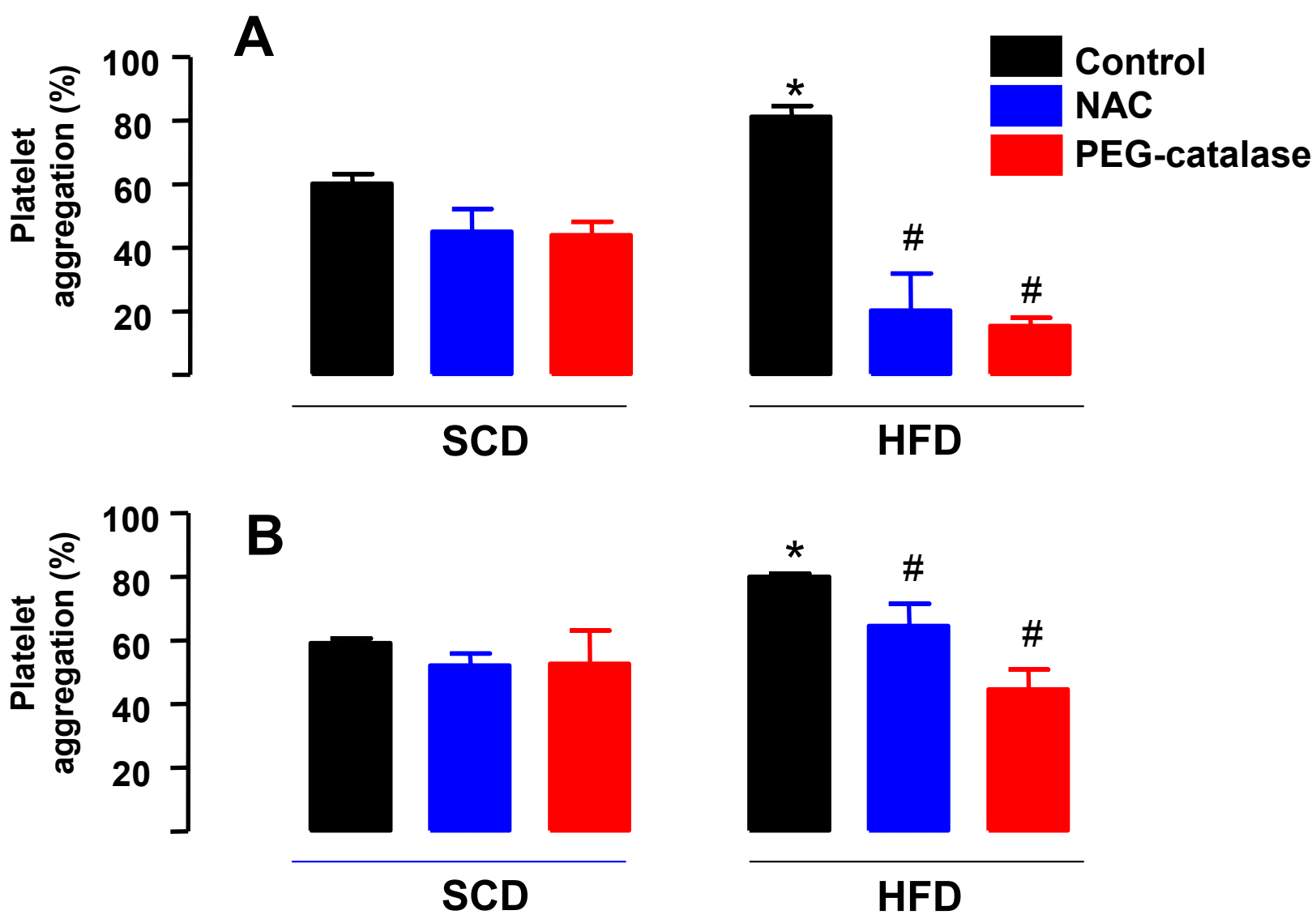

Figure 3 Effect of N-Acetylcysteine (NAC) and PEG-catalase on washed platelet aggregation of rats fed with a standard chow diet (SCD) or high-fat diet (HFD) during 10 weeks. Washed platelets $\left(1.2 \times 10^{8}\right.$ platelets $\left./ \mathrm{ml}\right)$ from SCD or HFD rats were stimulated with thrombin $(100 \mathrm{mU} / \mathrm{ml}$; panel A) or ADP $(10 \mu \mathrm{M}$; panel B) in the absence or the presence of NAC (1 mM) or PEG-catalase $(1000 \mathrm{U} / \mathrm{ml})$. Results are shown as mean \pm SEM values $(n=4-7) .{ }^{*} P<0.05$ compared with untreated control group. ${ }^{\#} P<0.05$ compared with the respective untreated platelets.

was prevented by the antioxidant compounds PEG-catalase and NAC in HFD group indicating a critical role for intracellular ROS in this phenomenon. Moreover, the NO donors SNP and SNAP, as well as the soluble guanylyl cyclase stimulator BAY 41-2272 showed a lower efficacy in inhibiting the platelet aggregation in HFD rats, possibly as a consequence of lower platelet cGMP productions in this diet-induced obesity model.

\section{Platelets, Hyperglycemia and Oxidative Stress}

Evidences have shown that persistent hyperglycemia can activate alternative glucose metabolism pathways, which in turn result in the formation of deleterious products derived from protein or lipid structure alterations named advanced glycation end products (AGEs), which can deeply affect the function of the cardiovascular system [16]. In vascular system, the interaction of AGES with their receptors (RAGE) can activate complex signaling pathways causing increased production of inflammatory mediators and massive ROS generation, resulting in reduced $\mathrm{NO}$ bioavailability and endothelium dysfunction [17], as well as alterations in coagulation system [18]. In cardiac tissues, the hyperglycemiainduced ROS activate the MEK/ERK pathway to increase GATA-4 phosphorylation, which in turn generates cardiac hypertrophy [19]. Hyperglycemia is also associated with dysregulation of sympathetic innervation to the myocardial tissues [20]. Thus, the mechanistic event by which diet-induced obesity causes platelet dysfunction may therefore be associated with hyperglycemia, which is consistent with the abnormality of the OGTT and ITT in HFD group. Previous studies show that acute hyperglycaemia enhances collagen-induced platelet aggregation via increased mitochondrial $\mathrm{O}_{2}{ }^{-}$production [21]. Acute hyperglycaemia following an oral glucose tolerance test or a carbohydrate-rich meal also promotes platelet activation in vivo [22,23].

Radicals derived from oxygen represent the most important class of ROS generated in living systems. Superoxide anion $\left(\mathrm{O}_{2}{ }^{-}\right)$is considered the primary ROS, and it can further interact with other molecules, either directly or through enzyme- or metal-catalyzed 

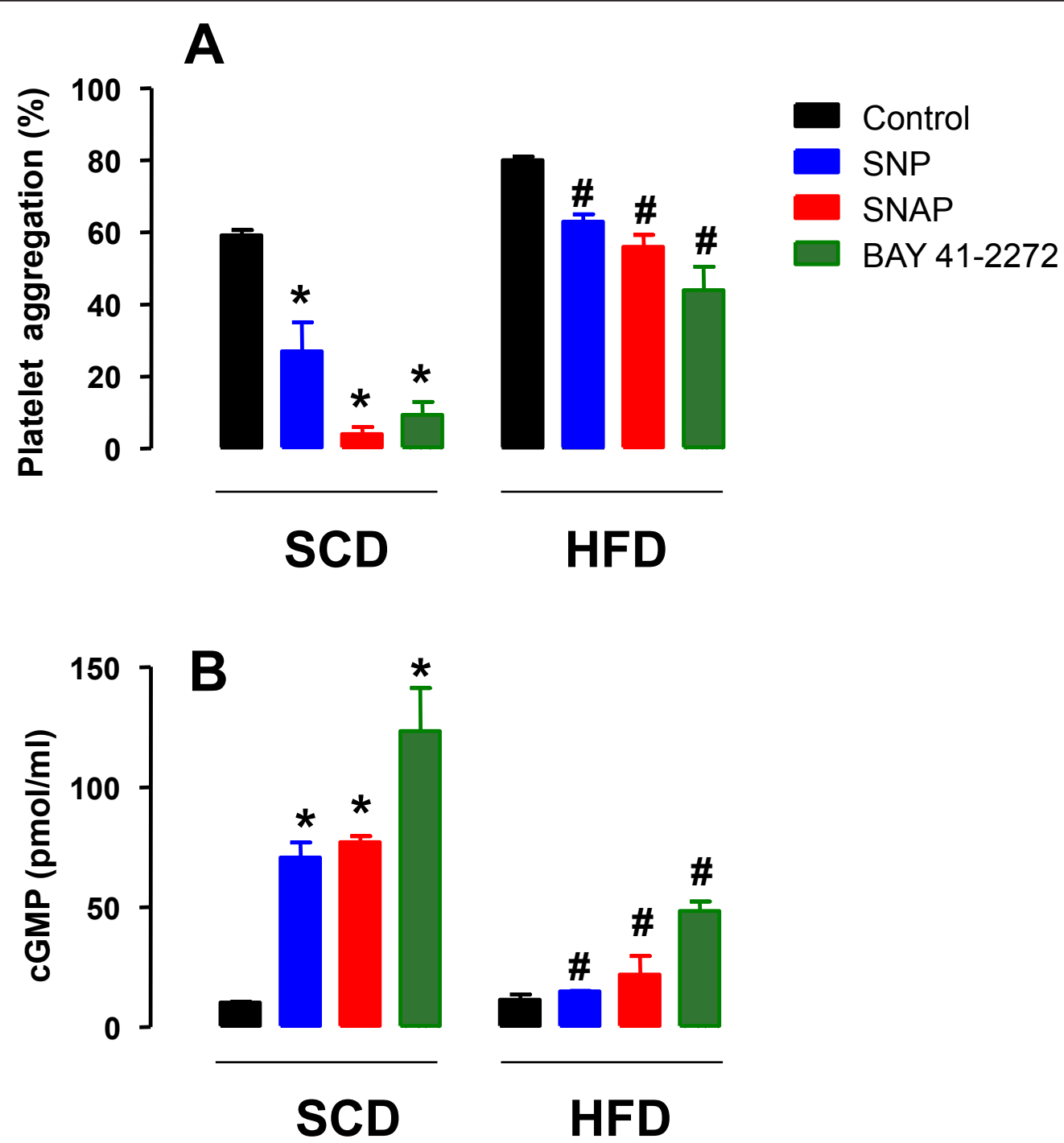

Figure 4 Effect of sodium nitroprusside (SNP), S-nitroso-N-acetylpenicillamine (SNAP) and BAY 41-2272 on washed platelet aggregation (panel A) and cyclic GMP production (panel B) in rats fed with a standard chow diet (SCD) or high-fat diet (HFD) during 10 weeks. Washed platelets $\left(1.2 \times 10^{8}\right.$ platelets $\left./ \mathrm{ml}\right)$ from SCD or HFD rats were stimulated with ADP $(10 \mu M)$ in the absence or the presence of SNP, SNAP or BAY 41-2272 (10 $\mu \mathrm{M}$ each). Results are shown as mean \pm SEM values $(n=4-7)$. ${ }^{*} P<0.05$ compared with untreated control group. ${ }^{\#} P<0.05$ compared with the respective agent in control group.

processes, to generate other physiological relevant ROS such as hydrogen peroxyde $\left(\mathrm{H}_{2} \mathrm{O}_{2}\right)$ and ${ }^{-} \mathrm{OH}$, as well as peroxynitrite $\left(\mathrm{ONOO}^{-}\right)$[24]. Adiposity in humans is reported to increase the risk of athero-thrombotic events due partly to increased oxidative stress, as evaluated by measurement of systemic biomarkers such as serum levels of lipid peroxidation, TNF- $\alpha$, free fatty acids and oxidized LDL [12]. Different sources, including platelets, may generate $\mathrm{O}_{2}{ }^{-}$including the NADPHoxidase, xanthine oxidase and arachidonate-derived prostaglandin-like metabolites [25-29]. However, the contribution of intraplatelet ROS in modulating platelet reactivity in conditions of adiposity has not been explored. Therefore, this study was designed to explore the ex-vivo washed platelet aggregation in HFD rats, and the potential role of intraplatelet ROS production and NO bioavailability in modulating platelet reactivity. Our data showed that ADP- and thrombin-induced platelet aggregation were significantly higher in HFD group, which was accompanied by higher levels of ROS production, as assessed by fluorescence assays using DCFH [30]. Moreover, prior incubation of platelets with the ROS scavengers PEG-catalase or NAC suppressed both the increased ROS production and the hyperaggregability in HFD rats. Altogether, our data indicate that ex vivo platelet hyperaggregability in HFD rats is 
Table 2 Effect of the prostacyclin analogue iloprost $(1 \mu \mathrm{M})$ in the ex vivo ADP-induced washed platelet aggregation

\begin{tabular}{lll}
\hline & SCD & HFD \\
\hline Control & $54.2 \pm 2.9 \%$ & $70.7 \pm 4.9 \%^{\#}$ \\
lloprost & $5.5 \pm 1.5 \% *$ & $5.7 \pm 2.2 \%{ }^{*}$ \\
\hline
\end{tabular}

Male Wistar rats were fed with either a standard chow diet $(S C D, n=4)$ or high-fat diet (HFD, $n=4$ ) during 10 weeks.

${ }^{*} P<0.05$ compared with respective untreated platelets. ${ }^{*} P<0.05$ compared with untreated platelets in SCD group.

closely linked with enhanced intraplatelet ROS production. A recent study showed that NAC, at concentrations attainable with oral dosing [31], significantly reduces ADP- and thrombin-induced platelet aggregation in whole blood of type 2 diabetic patients that is associated with an enhancement of its antioxidant activity [32].

Increased oxidative stress may also influence platelet function by decreasing NO bioavailability [12]. Nitric oxide is a ROS involved in multiple biological functions essential for the cardiovascular system and platelet function. Accordingly, in our study the ADP-induced platelet aggregation was markedly reduced by the NO donors, SNP and SNAP, in SCD rats, that was accompanied by marked elevations in the cGMP levels, as expected. Interestingly, in HFD rats, platelets were resistant to the cGMP elevations in response to SNP and SNAP, as well as to the inhibitory actions of these agents on platelet aggregation. It is likely that excess of $\mathrm{O}_{2}{ }^{-}$production in platelets of HFD rats inactivates SNP- and SNAPderived NO. This is consistent with studies performed in obese subjects and type 2 diabetic obese patients where platelets are resistant to glyceryl nitrate and SNP $[33,34]$.

\section{Platelet Hyperaggregability and Role of the Cyclic Nucleotides}

The soluble guanylyl cyclase (sGC) is a widely distributed signal transduction enzyme that, under activation by NO, converts GTP into the second messenger cGMP which in turn affects various downstream targets such as protein kinases, cyclic nucleotide-gated channels or phosphodiesterases [35]. One of the crucial pre-requisites of the NO-mediated sGC activation is the presence of the reduced haem moiety where its oxidation or loss renders the enzyme insensitive to NO. Nitric oxideindependent sGC activators have emerged as valuable tools to elucidate the physiopathology of the NO-sGCcGMP signaling pathway [36]. The compound BAY 412272 was reported as a haem-dependent and potent NO-independent sGC stimulator [37]. BAY 41-2272 directly stimulates sGC and increases the sensitivity of the enzyme to NO, generating significant amounts of
cGMP by stimulating the sGC mostly via NO-independent mechanisms [38,39]. Through this mechanism, BAY 41-2272 produces a variety of effects, including anti-aggregatory effects. In our study, BAY 41-2272 greatly elevated the cGMP levels and nearly abolished the platelet aggregation in SCD rats, as expected. However, the elevations of cGMP and inhibition of platelet aggregation by BAY 41-2272 in HFD rats were significantly lower compared with SCD group. This apparently indicates that $\mathrm{sGC}$ of platelets from HFD rats display a defect in producing appropriate amounts of intracellular cGMP. In rat platelets, under physiological conditions, inhibition of platelet aggregation by BAY 41-2272 requires the reduced form of $\mathrm{sGC}$ and the presence of NO [40]. Furthermore, the free radical $\mathrm{ONOO}^{-}$is able to oxidize the prosthetic haem group of sGC to its NOinsensitive $\mathrm{Fe}^{3+}$ state [41-43]. If that takes place in platelets from HFD rats, then BAY 41-2272 would be indeed expected to be less effective in activating sGC. In this aspect, it would be worth trying haem-independent sGC activators such as HMR1766 and BAY 58-2667 because they prevent sGC from oxidation-induced degradation, as evidenced in chinese hamster ovary cell line and in primary porcine endothelial cells [44]. Interestingly, the direct sGC activator HMR1766 has been shown to enhance the NO/cGMP-mediated signaling in platelets from streptozotocin-induced diabetic rats, reducing platelet-aggregates with other blood cells [45].

Besides the NO - cGMP - PDE5 pathway, the activation of platelets is inhibited by cAMP-elevating agents [46]. Elevation of intracellular cAMP levels can be achieved through the activation of adenylate cyclase either directly or through appropriately coupled membrane receptors, as well as by preventing the hydrolysis of cAMP by the cyclic nucleotide phosphodiesterases. In our study, the cAMP-elevating agent iloprost (stable prostacyclin analogue) suppressed the ADP-induced platelet aggregation in both SCD and HFD groups, excluding that hyperaggregability in HFD rats reflect changes in the cAMP signaling pathway.

\section{Conclusions}

Our findings clearly show that metabolic abnormalities as consequence of HFD in rats cause platelet hyperaggregability involving enhanced intraplatelet ROS production and decreased NO bioavailability accompanied by potential defects in the prosthetic haem group of sGC.

List of abbreviations

ADP: adenosine 5'diphosphate; HFD: high fat diet; HFF: high fat-fed; $\mathrm{H}_{2} \mathrm{O} 2$ : hydrogen peroxyde; IBMX: 3-isobutyl-I-methyl-xanthine; ITT: insulin tolerance test; NAC: N-acetylcysteine; NO: nitric oxide; OGTT: oral glucose tolerance test; ONOO-: peroxynitrite; PRP: Platelet-rich plasma; ROS: reactive-oxygen species; sGC: soluble guanylyl cyclase; SNAP: S-nitroso-N-acetylpenicillamine; O2-: superoxide anion; TXA2: thromboxane A2. 


\section{Acknowledgements}

Authors thank Fundação de Amparo à Pesquisa do Estado de São Paulo (FAPESP) and Conselho Nacional de Desenvolvimento Científico e Tecnológico (CNPq) for financial support.

\section{Author details}

'Department of Pharmacology, Faculty of Medical Sciences, University of Campinas (UNICAMP), Campinas, Sao Paulo, Brazil. ${ }^{2}$ Department of Physical Education, Institute of Bioscience, UNESP, Rio Claro, Sao Paulo, Brazil.

\section{Authors' contributions}

$\mathrm{AZ}$ and EA carried out interpreting the data and writing the manuscript; PFM, RPM, MAD, MEC, MELP and SM carried out data acquisition and reviewing statistical analysis. All authors read and approved the final manuscript.

\section{Competing interests}

The authors declare that they have no competing interests.

Received: 2 December 2011 Accepted: 16 January 2012 Published: 16 January 2012

\section{References}

1. Andrews RK, Berndt MC: Platelet physiology and thrombosis. Thromb Res 2004, 114:447-53.

2. Denis CV, Wagner DD: Platelet adhesion receptors and their ligands in mouse models of thrombosis. Arterioscler Thromb Vasc Biol 2007, 27:728-39.

3. Jackson SP: The growing complexity of platelet aggregation. Blood 2007 109:5087-95

4. Varga-Szabo D, Pleines I, Nieswandt B: Cell adhesion mechanisms in platelets. Arterioscler Thromb Vasc Biol 2008, 28:403-12.

5. Wei AH, Schoenwaelder SM, Andrews RK, Jackson SP: New insights into the haemostatic function of platelets. Br J Haematol 2009, 147:415-30.

6. Kroll MH, Hellums JD, McIntire LV, Schafer Al, Moake JL: Platelets and shear stress. Blood 1996, 88:1525-41.

7. Schäfer A, Bauersachs J: Endothelial dysfunction, impaired endogenous platelet inhibition and platelet activation in diabetes and atherosclerosis. Curr Vasc Pharmacol 2008, 6:52-60.

8. Rivera J, Lozano ML, Navarro-Núñez L, Vicente V: Platelet receptors and signaling in the dynamics of thrombus formation. Haematologica 2009, 94:700-711.

9. Libby P, Simon DI: Inflammation and thrombosis: the clot thickens. Circulation 2001, 103:1718-1720

10. Packard RR, Libby P: Inflammation in atherosclerosis: from vascular biology to biomarker discovery and risk prediction. Clin Chem 2008, 54:24-38.

11. De Caterina R, Massaro M, Scoditti E, Annunziata Carluccio M: Pharmacological modulation of vascular inflammation in atherothrombosis. Ann N Y Acad Sci 2010, 1207:23-31.

12. Anfossi G, Russo I, Trovati M: Platelet dysfunction in central obesity. Nutr Metab Cardiovasc Dis 2009, 19:440-449.

13. Kilkenny C, Browne WJ, Cuthill IC, Emerson M, Altman DG: Improving bioscience research reporting: the ARRIVE guidelines for reporting animal research. PloS Biol 2010, 8:1000412.

14. Calixto MC, Lintomen L, Schenka A, Saad MJ, Zanesco A, Antunes E: Obesity enhances eosinophilic inflammation in a murine model of allergic asthma. Br J Pharmacol 2010, 159:617-625.

15. Possel H, Noack H, Auqustin W, Keilhoff G, Wolf G: 2,7Dihydrodichlorofluorescein diacetate as a fluorescent marker for peroxynitrite formation. FEBS Lett 1997, 416:175-178.

16. Grillo MA, Colombatto S: Advanced glycation end-products (AGEs): involvement in aging and in neurodegenerative diseases. Amino Acids 2008, 35:29-36

17. Goldin A, Beckman JA, Schmidt AM, Creager MA: Advanced glycation end products: sparking the development of diabetic vascular injury. Circulation 2006, 114:597-605.

18. Won SJ, Tang XN, Suh SW, Yenari MA, Swanson RA: Hyperglycemia promotes tissue plasminogen activator-induced hemorrhage by Increasing superoxide production. Ann Neurol 2011, 70(4):583-590.
19. Ku PM, Chen LJ, Liang JR, Cheng KC, Li YX, Cheng JT: Molecular role of GATA binding protein 4 (GATA-4) in hyperglycemia-induced reduction of cardiac contractility. Cardiovasc Diabetol 2011, 10:57.

20. Thackeray JT, Radziuk J, Harper ME, Suuronen EJ, Ascah KJ, Beanlands RS, Dasilva JN: Sympathetic nervous dysregulation in the absence of systolic left ventricular dysfunction in a rat model of insulin resistance with hyperglycemia. Cardiovasc Diabetol 2011, 10:75.

21. Yamagishi SI, Edelstein D, Du XL, Brownlee M: Hyperglycemia potentiates collagen-induced platelet activation through mitochondrial superoxide overproduction. Diabetes 2001, 50:1491-1494.

22. Yngen M, Ostenson CG, Li N, Hjemdahl P, Wallén NH: Acute hyperglycemia increases soluble P-selectin in male patients with mild diabetes mellitus. Blood Coagul Fibrinolysis 2001, 12:109-116.

23. Yngen M, Ostenson CG, Hjemdahl P, Wallén NH: Meal-induced platelet activation in Type 2 diabetes mellitus: effects of treatment with repaglinide and glibenclamide. Diabet Med 2006, 23:134-140

24. Gresele P, Cerletti C, Guglielmini G, Pignatelli P, de Gaetano G, Violi F: Effects of resveratrol and other wine polyphenols on vascular function: an update. J Nutr Biochem 2011, 22:201-211.

25. Seno T, Inoue N, Gao D, Okuda M, Sumi Y, Matsui K, Yamada S, Hirata Kl, Kawashima S, Tawa R, Imajoh-Ohmi S, Sakurai H, Yokoyama M: Involvement of NADH/NADPH oxidase in human platelet ROS production. Thromb Res 2001, 103:399-409.

26. Wachowicz B, Olas B, Zbikowska HM, Buczyński A: Generation of reactive oxygen species in blood platelets. Platelets 2002, 13:175-182.

27. Pignatelli P, Sanguigni V, Lenti L, Ferro D, Finocchi A, Rossi P, Violi F: gp91phox-dependent expression of platelet CD40 ligand. Circulation 2004, 110:1326-1329

28. Begonja AJ, Gambaryan S, Geiger J, Aktas B, Pozgajova M, Nieswandt B, Walter U: Platelet $\mathrm{NAD}(\mathrm{P}) \mathrm{H}$-oxidase-generated ROS production regulates alphallbbeta3-integrin activation independent of the NO/cGMP pathway. Blood 2005, 106:2757-2560

29. Arthur JF, Gardiner EE, Kenny D, Andrews RK, Berndt MC: Platelet receptor redox regulation. Platelets 2008, 19:1-8.

30. Myhre O, Andersen JM, Aarnes H, Fonnum F: Evaluation of the probes $2^{\prime}, 7^{\prime}$-dichlorofluorescin diacetate, luminol, and lucigenin as indicators of reactive species formation. Biochem Pharmacol 2003, 65:1575-1582.

31. Gibson KR, Neilson IL, Barrett F, Winterburn TJ, Sharma S, MacRury SM, Megson IL: Evaluation of the antioxidant properties of $\mathrm{N}$-acetylcysteine in human platelets: prerequisite for bioconversion to glutathione for antioxidant and antiplatelet activity. J Cardiovasc Pharmacol 2009, 54:319-326.

32. Gibson KR, Winterburn TJ, Barrett F, Sharma S, MacRury SM, Megson IL: Therapeutic potential of $\mathrm{N}$-acetylcysteine as an antiplatelet agent in patientswith type-2 diabetes. Cardiovasc Diabetol 2011, 10:43-51.

33. Anfossi G, Mularoni EM, Burzacca S, Ponziani MC, Massucco P, Mattiello L, Cavalot $F$, Trovati M: Platelet resistance to nitrates in obesity and obese NIDDM, and normal platelet sensitivity to both insulin and nitrates in lean NIDDM. Diabetes Care 1998, 21:121-126.

34 Anfossi G, Russo I, Massucco P, Mattiello L, Doronzo G, De Salve A, Trovati M: Impaired synthesis and action of antiaggregating cyclic nucleotides in platelets from obese subjects: possible role in platelet hyperactivation in obesity. Eur J Clin Invest 2004, 34:482-489.

35. Francis SH, Busch JL, Corbin JD, Sibley D: cGMP-dependent protein kinases and CGMP phosphodiesterases in nitric oxide and cGMP action. Pharmacol Rev 2010, 62:525-63.

36. Stasch JP, Hobbs AJN: NO-independent, haem-dependent soluble guanylate cyclase stimulators. Handb Exp Pharmacol 2009, 191:277-308.

37. Stasch JP, Becker EM, Alonso-Alija C, Apeler H, Dembowsky K, Feurer A, Gerzer R, Minuth T, Perzborn E, Pleiss U, Schröder H, Schroeder W, Stahl E, Steinke W, Straub A, Schramm M: NO-independent regulatory site on soluble guanylate cyclase. Nature 2001, 410:212-215.

38. Stasch JP, Alonso-Alija C, Apeler H, Dembowsky K, Feurer A, Minuth T, Perzborn E, Schramm M, Straub A: Pharmacological actions of a novel NO-independent guanylyl cyclase stimulator, BAY 41-8543: in vitro studies. Br J Pharmacol 2002, 135:333-343.

39. Evgenov OV, Pacher P, Schmidt PM, Haskó G, Schmidt HH, Stasch JPN: NOindependent stimulators and activators of soluble guanylate cyclase: discovery and therapeutic potential. Nat Rev Drug Discov 2006, 5:755-678

40. Roger S, Badier-Commander C, Paysant J, Cordi A, Verbeuren TJ, Félétou M: The anti-aggregating effect of BAY 41-2272, a stimulator of soluble 
guanylyl cyclase, requires the presence of nitric oxide. Br J Pharmacol 2010, 161:1044-1058.

41. Gladwin MT: Deconstructing endothelial dysfunction: soluble guanylyl cyclase oxidation and the NO resistance syndrome. J Clin Invest 2006, 116:2330-2332.

42. Stasch JP, Schmidt PM, Nedvetsky PI, Nedvetskaya TY, Arum Kumar HS, Meurer S, Taye A, Knorr A, Lapp H, Müller H, Turgay Y, Rothkegel C, Tersteegen A, Kemp-Harper B, Müller-Esterl W, Schmidt HH: Targeting the heme-oxidized nitric oxide receptor for selective vasodilatation of diseased blood vessels. J Clin Invest 2006, 116:2552-2561.

43. Chirkov YY, Horowitz JD: Impaired tissue responsiveness to organic nitrates and nitric oxide: a new therapeutic frontier? Pharmacol Ther 2007, 116:287-305.

44. Hoffmann LS, Schmidt PM, Keim Y, Schaefer S, Schmidt HH, Stasch JP: Distinct molecular requirements for activation or stabilization of soluble guanylyl cyclase upon haem oxidation-induced degradation. $\mathrm{Br} J$ Pharmacol 2009, 157:781-795.

45. Schäfer A, Flierl U, Kobsar A, Eigenthaler M, Ertl G, Bauersachs J: Soluble guanylyl cyclase activation with HMR1766 attenuates platelet activation in diabetic rats. Arterioscler Thromb Vasc Biol 2006, 26:2813-2818.

46. Noé L, Peeters K, Izzi B, Van Geet C, Freson K: Regulators of platelet cAMP levels: clinical and therapeutic implications. Curr Med Chem 2010, 17:2897-2905

doi:10.1186/1475-2840-11-5

Cite this article as: Monteiro et al.: Platelet hyperaggregability in highfat fed rats: A role for intraplatelet reactive-oxygen species production. Cardiovascular Diabetology 2012 11:5.

\section{Submit your next manuscript to BioMed Central} and take full advantage of:

- Convenient online submission

- Thorough peer review

- No space constraints or color figure charges

- Immediate publication on acceptance

- Inclusion in PubMed, CAS, Scopus and Google Scholar

- Research which is freely available for redistribution

Submit your manuscript at www.biomedcentral.com/submit
Biomed Central 